\title{
Beam halo studies using a three-dimensional particle-core model
}

\author{
Ji Qiang and Robert D. Ryne \\ Los Alamos National Laboratory, Los Alamos, New Mexico 87545
}

(Received 16 July 1999; published 22 June 2000)

\begin{abstract}
In this paper we present a study of beam halo based on a three-dimensional particle-core model of an ellipsoidal bunched beam in a constant focusing channel including the effects of nonlinear rf focusing. For an initially mismatched beam, three linear envelope modes - a high frequency mode, a low frequency mode, and a quadrupole mode - are identified for an azimuthally symmetric bunched beam. The high frequency mode has three components all in phase; the low frequency mode has the transverse components in phase and the longitudinal component $180^{\circ}$ out of phase; the quadrupole mode has no longitudinal component, and the two transverse components in the mode are $180^{\circ}$ out of phase. We also study the case of an ellipsoidal bunched beam without azimuthal symmetry and find that the high frequency mode and the low frequency mode are still present but the quadrupole mode is replaced by a new mode with transverse components $180^{\circ}$ out of phase and a nonzero longitudinal component. Previous studies, which generally addressed the situation where the longitudinal-to-transverse focusing strength is roughly 0.6 or less, conclude that the oscillation of the high frequency mode is predominantly transverse, and that of the low frequency mode is predominantly longitudinal. In this paper we present a systematic study of the features of the modes as a function of the longitudinal-to-transverse focusing strength ratio. We find that, when the ratio is greater than unity, the high frequency mode may contain a significant longitudinal component. Thus, excitation of the high frequency mode in this situation can be responsible for the formation of longitudinal beam halo. Furthermore, while previous studies have observed halo amplitudes roughly 2-3 times the matched beam edge, for the present parameters we observe much larger amplitudes ( 5 times or more). This is due to the fact that the longitudinalto-transverse focusing ratio used here is greater than that of previous studies. The finding of large transverse halo amplitude can have significant impact on the design of high-intensity ion accelerators where the longitudinal-to-transverse focusing ratio is slightly greater than unity in some parts of the linac.
\end{abstract}

PACS numbers: 41.75.-i, , 29.27.Bd

\section{INTRODUCTION}

High-intensity ion linacs have been proposed in recent years for applications such as the driver for a spallation neutron source, the production of tritium, and the transmutation of radioactive waste. These high-intensity linacs put strong limits on beam loss to the wall of the beam pipe, since small fractional losses in a high average-current machine can cause unacceptably high levels of radioactivation. An important mechanism for beam loss is that due to the presence of a large amplitude, low-intensity beam halo far from the beam core. Such halos have been observed in existing machines, such as the LANSCE proton linac [1]. Furthermore, the evolution of beam halo has been studied in experiments at the University of Maryland [2]. In the design of next-generation accelerators, it is very important to understand the physics of the beam halo.

The physics of beam halo has been extensively studied through analytical theory and multiparticle simulations [3-15]. In these studies, the particle-core model has been frequently used due to its usefulness in understanding the essential mechanism of halo formation and helping to predict the extent of beam halo. In this approach, the beam core is modeled using rms envelope equations, and the core oscillates due to an initial mismatch. Assuming that the oscillating beam has a prescribed density profile, the dynamics of halo particles are modeled by studying the motion of test particles subject to the external focusing force and the time-dependent, nonlinear space-charge field associated with the core. The test particles do not affect the motion of the core, which is consistent with the assumption that the halo represents only a small fraction of the total beam charge. The oscillation frequency of test particles is amplitude dependent due to the nonlinear space-charge force from the core. This results in some particles being driven to large amplitudes through a parametric resonance with the core [4].

In early studies of beam halo using the particle-core model, a one-dimensional model was used to study the transverse halo dynamics for a round infinitely long beam propagating through a continuous focusing or a periodic focusing channel $[6-8,10,13,16]$. Two-dimensional $(r-z)$ particle-core models with nonlinear rf fields have also been used to study the longitudinal beam halo for an isolated ellipsoidal bunched beam and the coupling between the transverse and longitudinal halo $[17,18]$. Three-dimensional models with linear $\mathrm{rf}$ focusing have been employed to study the halo formation of a bunched beam $[19,20]$. In Ref. [19], Bongardt obtained the envelope mode frequencies using an approximate analytical 
TABLE I. Physical parameters in the test example.

\begin{tabular}{lc}
\hline \hline Bunch current (A) & 0.1 \\
Proton energy (MeV) & 471.4 \\
Synchronous phase (degrees) & -30 \\
rf frequency (MHz) & 700 \\
Accelerating gradient $(\mathrm{MV} / \mathrm{m})$ & 5.246 \\
Lattice period $(\mathrm{m})$ & 8.54 \\
Transverse phase advance without current (degrees) & 81 \\
Longitudinal phase advance without current & \\
$\quad$ (degrees) & 83.51 \\
Transverse phase advance with current (degrees) & 47.6 \\
Longitudinal phase advance with current (degrees) & 69.8 \\
Transverse unnormalized rms emittance & \\
$\quad(\pi$ mm mrad) & 0.2319 \\
Longitudinal unnormalized rms emittance & \\
$\quad(\pi$ mm mrad) & 0.4750 \\
Transverse matched edge size (mm) & 1.543 \\
Longitudinal matched edge size $(\mathrm{mm})$ & 1.825 \\
\hline \hline
\end{tabular}

formula, and, in Ref. [20], Pichoff presented an exact calculation of the envelope mode frequencies for a bunched beam with azimuthal symmetry. In this paper, we will use a fully three-dimensional particle-core model to study all the modes of oscillation of an ellipsoidal bunched beam in a constant focusing channel, and possible beam halo formation. Three envelope modes will be identified and their effects on the formation of beam halo through a parametric resonance with test particles will be studied. Our analysis also includes the effect of nonlinear rf fields. The physical parameters for the present study are shown in Table I. For this study, we have chosen a zero-current, longitudinal-to-transverse focusing strength ratio of 1.03. This ratio, which is higher than that of conventional designs, is relevant for future superconducting linacs which may operate with high accelerating gradients. The present study uses a $100 \mathrm{~mA}$ beam with a longitudinal rms emittance of about twice the transverse emittance. The remaining physical parameters are similar to a section of the design of the accelerator production of tritium linac [21]. Though our analysis is fully three-dimensional, the numerical results based on Table I have azimuthal symmetry.

The organization of this paper is as follows: the threedimensional particle-core model is described in Sec. II, the linear envelope modes are discussed in Sec. III, the test particle dynamics under the three envelope modes are presented in Sec. IV, and the conclusions are summarized in Sec. V.

\section{THREE-DIMENSIONAL PARTICLE-CORE MODEL}

In the particle-core model, the system consists of a beam core and test particles. The core, which contains most of the particles, is modeled by the rms envelope equations. The test particles contain a small fraction of the beam and are subject to the effects of external forces and space charge forces due to the core. The physical origin of these particles is beyond the scope of the particle-core model. However, they could be present in the tail of the initial distribution or they could be generated by instabilities of the core $[8,22]$. The effects of test particles on the core and the mutual Coulomb interactions among test particles are neglected. In our model the core is assumed to be an ellipsoid with a uniform charge density distribution. The boundary of core is defined by a three-dimensional elliptical equation as

$$
\frac{x^{2}}{r_{x}^{2}}+\frac{y^{2}}{r_{y}^{2}}+\frac{z^{2}}{r_{z}^{2}}=1,
$$

where the semiaxes $r_{i}$ are related to the rms beam sizes $a_{i}$ by $r_{i}=\sqrt{5} a_{i}, i=x, y, z$. Three-dimensional $\mathrm{rms}$ equations were derived in $[23,24]$, and the inclusion of nonlinear $\mathrm{rf}$ focusing was treated in [17]. Under the smooth approximation, the envelope equations for the bunched beam including nonlinear rf focusing are given by

$$
\begin{gathered}
\frac{d^{2} r_{x}}{d z^{2}}+k_{x 0}^{2} r_{x}-I_{x}\left(r_{x}, r_{y}, r_{z}, 0\right) r_{x}-\frac{\epsilon_{x}^{2}}{r_{x}^{3}}=0 \\
\frac{d^{2} r_{y}}{d z^{2}}+k_{y 0}^{2} r_{y}-I_{y}\left(r_{x}, r_{y}, r_{z}, 0\right) r_{y}-\frac{\epsilon_{y}^{2}}{r_{y}^{3}}=0 \\
\frac{d^{2} r_{z}}{d z^{2}}+k_{z 0}^{2} f\left(r_{z}\right) r_{z}-I_{z}\left(r_{x}, r_{y}, r_{z}, 0\right) r_{z}-\frac{\epsilon_{z}^{2}}{r_{z}^{3}}=0,
\end{gathered}
$$

with

$$
\begin{aligned}
& I_{i}\left(r_{x}, r_{y}, r_{z}, s\right) \\
& \quad=C \int_{s}^{\infty} \frac{d t}{\left(e_{i}^{2}+t\right) \sqrt{\left(r_{x}^{2}+t\right)\left(r_{y}^{2}+t\right)\left(\gamma^{2} r_{z}^{2}+t\right)}},
\end{aligned}
$$

where $e_{i}=r_{x}, r_{y}, \gamma r_{z}, \quad$ for $i=x, y, z$, and $C=$ $\frac{1}{2} \frac{3}{4 \pi \epsilon_{0}} \frac{q}{m c^{2}} \frac{I}{f_{r f} \beta^{2} \gamma^{2}}$. Here, $\epsilon_{0}$ is the vacuum permeability, $q$ is the charge, $m c^{2}$ is the rest energy of the particles, $c$ is the vacuum light speed, $I$ is the average beam current, $f_{\mathrm{rf}}$ is the $\mathrm{rf}$ bunch frequency, $\beta=v / c$, $v$ is the bunch speed, and $\gamma=1 / \sqrt{1-\beta^{2}}$. The quantities $k_{x 0}$ and $k_{y 0}$ in the envelope equations are the transverse betatron wave numbers at zero current, which are defined as $k_{i 0}=\sigma_{i 0} / L, i=x, y$, under the smooth approximation for a periodic quadrupole focusing array element. Here, $\sigma_{i 0}$ is the zero-current transverse phase advance per focusing period $L$. The longitudinal synchrotron wave number at zero current, $k_{z 0}$, is defined as $k_{z 0}=\sqrt{2 \pi q E_{0} T \sin \left(-\phi_{s}\right) /\left(\gamma^{3} \beta^{3} m c^{2} \lambda\right)}$, where $E_{0} T$ is the accelerating gradient, $\phi_{s}$ is the synchronous phase, and $\lambda$ is the rf wavelength. The function $f\left(r_{z}\right)$ in the 
envelope equation is a nonlinear $\mathrm{rf}$ focusing factor defined by

$$
\begin{aligned}
f\left(r_{z}\right)=\frac{15}{\left(k_{\eta} r_{z}\right)^{5}} & {\left[\left(3-\left(k_{\eta} r_{z}\right)^{2}\right) \sin \left(k_{\eta} r_{z}\right)\right.} \\
& \left.-3 k_{\eta} r_{z} \cos \left(k_{\eta} r_{z}\right)\right],
\end{aligned}
$$

where $k_{\eta}=2 \pi / \beta \lambda$ [17]. In the above envelope equations, we have used a continuous sinusoidal wave to represent the average effect of the synchronous rf space harmonics in the rf gap and neglected the acceleration of the rf field. Normally, it is necessary to assume that the external forces are linear in order to derive equations for the second moments that do not involve higher moments. However, by assuming that the ellipsoidal beam has a uniform density distribution, as we have done, one can derive equations involving only second moments. For a space charge dominated beam, this uniform-density assumption is a reasonable approximation [13]. These equations differ from the linear envelope equations in Refs. [19] and [20] with the inclusion of a nonlinear multiplicative factor $f\left(r_{z}\right)$ in the longitudinal equation. This factor approaches unity as $k_{\eta} r_{z}$ goes to zero. This corresponds to small beam bunch size with respect to the rf wavelength $\lambda$. The emittances $\epsilon_{x}, \epsilon_{y}$, and $\epsilon_{z}$ are 5 times the corresponding rms emittances.

The equations of motion for a test particle in the presence of a uniformly charged core and constant external fields, neglecting acceleration, are given by

$$
\begin{aligned}
& \frac{d^{2} x}{d z^{2}}+k_{x 0}^{2} x-I_{x}\left(r_{x}, r_{y}, r_{z}, s\right) x=0, \\
& \frac{d^{2} y}{d z^{2}}+k_{y 0}^{2} y-I_{y}\left(r_{x}, r_{y}, r_{z}, s\right) y=0,
\end{aligned}
$$

$$
\begin{aligned}
& \frac{d^{2} \Delta z}{d z^{2}}-k_{\xi}\left[\cos \left(-k_{\eta} \Delta z+\phi_{s}\right)-\cos \left(\phi_{s}\right)\right]- \\
& I_{z}\left(r_{x}, r_{y}, r_{z}, s\right) \Delta z=0,
\end{aligned}
$$

where $k_{\xi}=q E_{0} T / m c^{2} \beta^{2} \gamma^{3}$, and where the parameter $s$ is zero for a particle inside the core and is determined from the root of equation

$$
\frac{x^{2}}{r_{x}^{2}+s}+\frac{y^{2}}{r_{y}^{2}+s}+\frac{\Delta z^{2}}{r_{z}^{2}+s}=1,
$$

for a particle outside the core.

The above envelope equations and test particle equations constitute the basis of the three-dimensional particle-core model with a uniform core. In the numerical calculation, it is more convenient to use dimensionless equations. Letting $r_{i}=r_{i 0} R_{i}, i=x, y, z$, where $r_{i 0}$ is the matched core size, and $t=k_{x 0} z$, we obtain the dimensionless envelope equations as

$$
\begin{gathered}
R_{x}^{\prime \prime}+R_{x}-\left(1-\eta_{x}^{2}\right) \frac{\hat{I}_{x}(0)}{R_{y} R_{z} \hat{I}_{x 0}}-\frac{\eta_{x}^{2}}{R_{x}^{3}}=0, \\
R_{y}^{\prime \prime}+\frac{k_{y 0}^{2}}{k_{x 0}^{2}}\left[R_{y}-\left(1-\eta_{y}^{2}\right) \frac{R_{x} \hat{I}_{y}(0)}{R_{y}^{2} R_{z} \hat{I}_{y 0}}-\frac{\eta_{y}^{2}}{R_{y}^{3}}\right]=0,
\end{gathered}
$$

$$
R_{z}^{\prime \prime}+\frac{k_{z 0}^{2}}{k_{x 0}^{2}}\left[f R_{z}-\left(f_{0}-\eta_{z}^{2}\right) \frac{R_{x} \hat{I}_{z}(0)}{R_{y} R_{z}^{2} \hat{I}_{z 0}}-\frac{\eta_{z}^{2}}{R_{z}^{3}}\right]=0,
$$

with

$$
\hat{I}\left(R_{x}, R_{y}, R_{z}, s\right)_{i}=\int_{s}^{\infty} \frac{d t}{\left.\left(1+E_{i}\right) \sqrt{(1+t)\left[1+t r_{x 0}^{2} R_{x}^{2} /\left(r_{y 0}^{2} R_{y}^{2}\right)\right]\left[1+t r_{x 0}^{2} R_{x}^{2} /\left(\gamma^{2} r_{z 0}^{2} R_{z}^{2}\right)\right.}\right]}
$$

where $E_{1}=1, E_{2}=r_{x 0}^{2} R_{x}^{2} / r_{y 0}^{2} R_{y}^{2}, E_{3}=r_{x 0}^{2} R_{x}^{2} / \gamma^{2} r_{z 0}^{2} R_{z}^{2}$, and the integrals $\hat{I}_{i 0}$ are calculated for a matched beam core, $R_{x}=R_{y}=R_{z}=1$. The tune depressions $\eta_{x}, \eta_{y}$, and $\eta_{z}$ are defined as

$$
\begin{gathered}
\eta_{x}^{2}=\frac{k_{x 0}^{2}-I_{x 0}}{k_{x 0}^{2}}, \\
\eta_{y}^{2}=\frac{k_{y 0}^{2}-I_{y 0}}{k_{y 0}^{2}}, \\
\eta_{z}^{2}=\frac{f_{0} k_{z 0}^{2}-I_{z 0}}{k_{z 0}^{2}},
\end{gathered}
$$

where $f_{0}$ is the rf nonlinear focusing factor calculated with the matched core size. These three tune depressions can be related to each other using the following expressions:

$$
\begin{aligned}
\frac{k_{y 0}^{2}}{k_{x 0}^{2}} \frac{1-\eta_{y}^{2}}{1-\eta_{x}^{2}} & =\frac{r_{x 0}^{2}}{r_{y 0}^{2}} \frac{\hat{I}_{y 0}}{\hat{I}_{x 0}}, \\
\frac{k_{z 0}^{2}}{k_{x 0}^{2}} \frac{f_{0}-\eta_{z}^{2}}{1-\eta_{x}^{2}} & =\frac{r_{x 0}^{2}}{\gamma^{2} r_{z 0}^{2}} \frac{\hat{I}_{z 0}}{\hat{I}_{x 0}} .
\end{aligned}
$$

For the equations of motion of test particles, we define the dimensionless variables as $X=x / r_{x 0}, Y=$ $y / r_{y 0}$, and $Z=\Delta z / \gamma r_{z 0}$. The dimensionless equations of motion are

$$
\begin{gathered}
X^{\prime \prime}+X-X\left(1-\eta_{x}^{2}\right) \frac{\hat{I}_{x}(s)}{R_{x} R_{y} R_{z} \hat{I}_{x 0}}=0, \\
Y^{\prime \prime}+Y \frac{k_{y 0}^{2}}{k_{x 0}^{2}}\left[1-\left(1-\eta_{y}^{2}\right) \frac{R_{x} \hat{I}_{y}(s)}{R_{y}^{3} R_{z} \hat{I}_{y 0}}\right]=0,
\end{gathered}
$$




$$
Z^{\prime \prime}-k_{\xi}\left[\cos \left(-k_{\eta} \gamma r_{z 0} Z+\phi_{s}\right)-\cos \left(\phi_{s}\right)\right] /\left(k_{x 0}^{2} \gamma r_{z 0}\right)-Z \frac{k_{z 0}^{2}}{k_{x 0}^{2}}\left(f_{0}-\eta_{z}^{2}\right) \frac{R_{x} \hat{I}_{z}(s)}{R_{y} R_{z}^{3} \hat{I}_{z 0}}=0,
$$

where the parameter $s$ in the integrals $\hat{I}_{i}, i=x, y, z$, is zero for particles inside the core and is the root of the following equation:

$$
\begin{aligned}
\frac{X^{2} / R_{x}^{2}}{1+s}+ & \frac{\left(Y^{2} / R_{x}^{2}\right)\left(r_{y 0}^{2} / r_{z 0}^{2}\right)}{\left(R_{y}^{2} / R_{x}^{2}\right)\left(r_{y 0}^{2} / r_{z 0}^{2}\right)+s}+ \\
& \frac{\left(Z^{2} / R_{x}^{2}\right)\left[\left(\gamma r_{z 0}\right)^{2} / r_{x 0}^{2}\right]}{\left(R_{y}^{2} / R_{x}^{2}\right)\left[\left(\gamma r_{z 0}\right)^{2} / r_{x 0}^{2}\right]+s}=1,
\end{aligned}
$$

for the particle outside the core. This group of coupled nonlinear ordinary differential equations is solved numerically using a leap-frog algorithm.

\section{LINEAR ENVELOPE MODES}

The steady state solution of the envelope equations has three components which define the stationary core size. If the initial size of the beam is the same as the stationary core size, the beam is said to be matched and the core size will stay constant. If there exists a difference between the initial beam core size and the stationary beam core size, the beam is said to be mismatched and, if the mismatch is small, three linear eigenmodes of the core envelope may be excited. To obtain the oscillation wave number of these modes, we have used the following linearized envelope equations:

$$
\begin{gathered}
\delta R_{x}^{\prime \prime}+\left[4 \eta_{x}^{2}+3\left(1-\eta_{x}^{2}\right) F_{x x} / \hat{I}_{x 0}\right] \delta R_{x}+\left(1-\eta_{x}^{2}\right) F_{x y} / \hat{I}_{x 0} \delta R_{y}+\left(1-\eta_{x}^{2}\right) F_{x z} / \hat{I}_{x 0} \delta R_{z}=0, \\
\delta R_{y}^{\prime \prime}+k_{y 0}^{2} / k_{x 0}^{2}\left\{\left[4 \eta_{y}^{2}+3\left(1-\eta_{y}^{2}\right) F_{y y} / \hat{I}_{y 0}\right] \delta R_{y}+\left(1-\eta_{y}^{2}\right) F_{x y} / \hat{I}_{y 0} \delta R_{x}+\left(1-\eta_{y}^{2}\right) F_{y z} / \hat{I}_{y 0} \delta R_{z}\right\}=0, \\
\delta R_{z}^{\prime \prime}+k_{z 0}^{2} / k_{x 0}^{2}\left\{\left[4 \eta_{z}^{2}+3\left(f_{0}-\eta_{z}^{2}\right) F_{z z} / \hat{I}_{z 0}+\frac{\partial f\left(r_{z 0}\right)}{\partial r_{z}} r_{z 0}\right] \delta R_{z}\right. \\
\left.+\left(f_{0}-\eta_{z}^{2}\right) F_{x z} / \hat{I}_{z 0} \delta R_{x}+\left(f_{0}-\eta_{z}^{2}\right) F_{y z} / \hat{I}_{z 0} \delta R_{y}\right\}=0,
\end{gathered}
$$

with

$$
\begin{aligned}
& F_{x x}=\int_{0}^{\infty} \frac{d t}{(1+t)^{5 / 2}\left(1+t r_{x 0}^{2} / r_{y 0}^{2}\right)^{1 / 2}\left(1+t r_{x 0}^{2} / \gamma^{2} r_{z 0}^{2}\right)^{1 / 2}}, \\
& F_{y y}=\int_{0}^{\infty} \frac{d t}{(1+t)^{1 / 2}\left(1+t r_{x 0}^{2} / r_{y 0}^{2}\right)^{5 / 2}\left(1+t r_{x 0}^{2} / \gamma^{2} r_{z 0}^{2}\right)^{1 / 2}}, \\
& F_{z z}=\int_{0}^{\infty} \frac{d t}{(1+t)^{1 / 2}\left(1+t r_{x 0}^{2} / r_{y 0}^{2}\right)^{1 / 2}\left(1+t r_{x 0}^{2} / \gamma^{2} r_{z 0}^{2}\right)^{5 / 2}}, \\
& F_{x y}=\int_{0}^{\infty} \frac{d t}{(1+t)^{3 / 2}\left(1+t r_{x 0}^{2} / r_{y 0}^{2}\right)^{3 / 2}\left(1+t r_{x 0}^{2} / \gamma^{2} r_{z 0}^{2}\right)^{1 / 2}}, \\
& F_{x z}=\int_{0}^{\infty} \frac{d t}{(1+t)^{3 / 2}\left(1+t r_{x 0}^{2} / r_{y 0}^{2}\right)^{1 / 2}\left(1+t r_{x 0}^{2} / \gamma^{2} r_{z 0}^{2}\right)^{3 / 2}}, \\
& F_{y z}^{\infty} \frac{d t}{(1+t)^{1 / 2}\left(1+t r_{x 0}^{2} / r_{y 0}^{2}\right)^{3 / 2}\left(1+t r_{x 0}^{2} / \gamma^{2} r_{z 0}^{2}\right)^{3 / 2}},
\end{aligned}
$$

where the mismatched envelope $R_{i}=R_{i 0}+\delta R_{i}, i=$ $x, y, z$, and $\delta R_{i} / R_{i 0} \ll 1$. For a matched beam, $R_{i 0}=$ 1.0. From the above linear ordinary differential equations, we can find the eigenmodes of mismatched core oscillation by using the ansatz $\delta R_{j} \sim \exp \left(i k_{j} t\right)$. For the physical parameters given in Table I, we get the normalized wave number 1.945 for the high frequency mode, 1.641 for the low frequency mode, and 1.456 for the quadrupole mode. As a verification of our calculation, we also computed the mode wave number through a Fourier analysis of mismatched envelope equations. Figure 1 shows the $t$-dependent envelopes for $R_{x}, R_{y}$, and $R_{z}$ with initial mismatch $\delta R_{x}=0.01, \delta R_{y}=-0.05$, and $\delta R_{z}=0.008$. The power spectra for the above envelopes are presented in Fig. 2. The normalized wave number for the high mode is 1.941, for the low mode it is 1.642 , and for the quadrupole mode it is 1.452 . These wave numbers agree very well with the calculations from the 


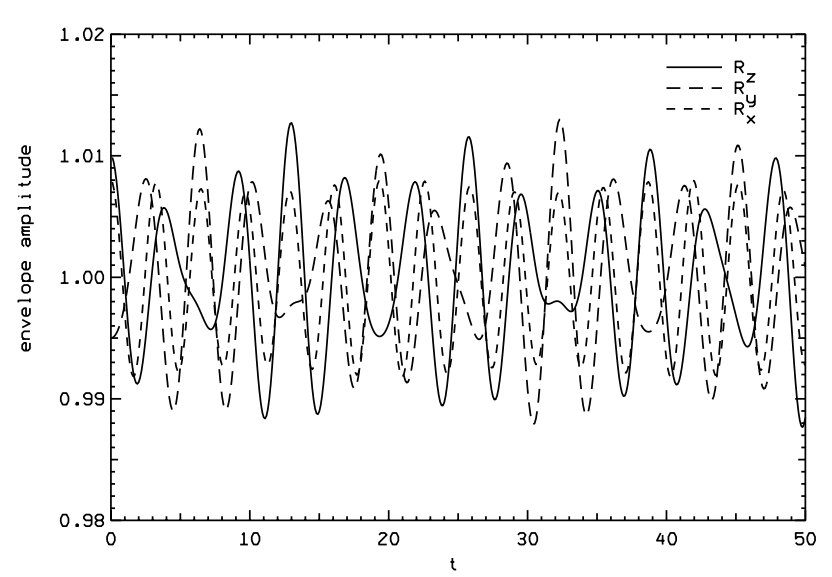

FIG. 1. The $t$-dependent envelopes for $R_{x}, R_{y}$, and $R_{z}$ with initial mismatch $\delta R_{x}=0.01, \quad \delta R_{y}=-0.05$, and $\delta R_{z}=0.008$.

linear eigenmode analysis. In Fig. 2 we also note that the quadrupole mode involves only transverse oscillation (i.e., $\delta R_{z}=0$ in the power spectrum around 1.452).

To investigate the possible resonance between test particles and the mismatched core oscillation, we calculated the ratio of the possible test particle wave numbers to the mismatched mode wave number as a function of current with all the other physical parameters as shown in Table I. The results for the transverse betatron motion are given in Fig. 3. It shows that a 1:2 resonance between a test particle and the low mode and quadrupole mode is always possible. For the high mode, the 1:2 resonance is possible with a current greater than $40 \mathrm{~mA}$. With increasing current, other higher-order resonances are also possible. At a current of $100 \mathrm{~mA}$, the 1:2 resonance between the betatron motion of test particles and all three mismatch modes is present. Figure 4 shows the ratio between the wave number of particle synchrotron motion and the

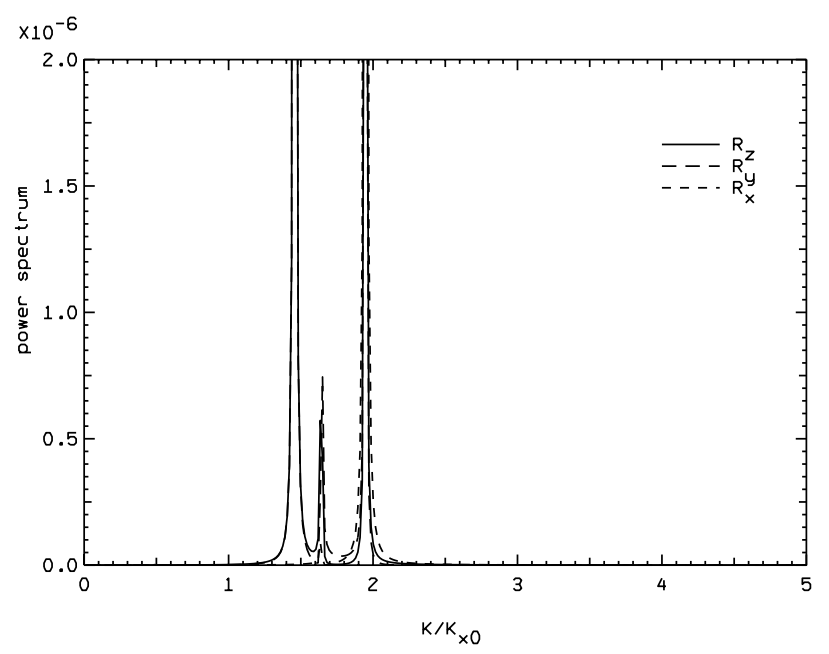

FIG. 2. The power spectra for the envelopes $R_{x}, R_{y}$, and $R_{z}$ with initial mismatch $\delta R_{x}=0.01, \delta R_{y}=-0.05$, and $\delta R_{z}=$ 0.008 .

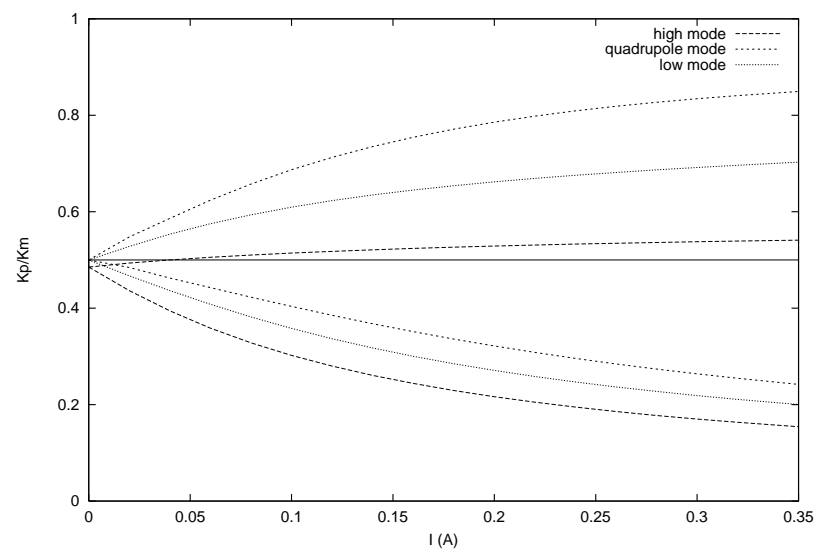

FIG. 3. The ratio of particle betatron wave number to mode wave number as a function of current.

wave number of the high mode and the low mode. Here, the 1:2 resonance with the high mode is excited when the current is greater than $60 \mathrm{~mA}$. The $1: 2$ resonance with the low mode is only present at currents greater than $230 \mathrm{~mA}$. This is different from some previous studies where the 1:2 resonance occurs regardless of the beam current. This is because, in the previous studies with linear focusing, the ratio of the test particle wave number to the envelope mode wave number always starts from 0.5 with zero current. For the nonlinear focusing, this ratio can be greater than 0.5 for zero current. This suggests that there exists some current region without the 1:2 resonance in the longitudinal plane, which is observed in this study and the study of Barnard et al. [17]. In Fig. 5, we show the ratio of relative amplitude of longitudinal oscillation to that of transverse oscillation, $\delta R_{z} / \delta R_{x}$, for the high mode and the low mode as a function of current. For the high mode, the oscillation is predominantly longitudinal at low current and is in phase with the transverse oscillation. Furthermore, the dominance decreases with increasing current. For the low mode, the oscillation

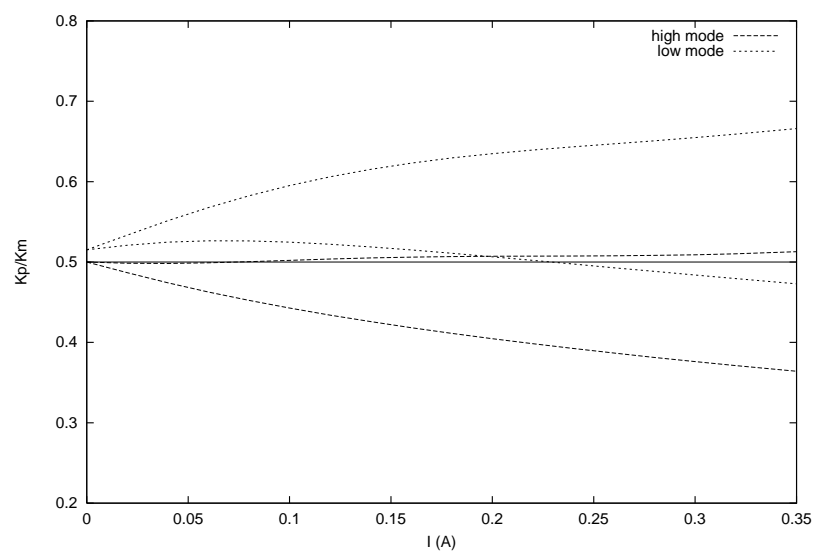

FIG. 4. The ratio between the wave number of particle synchrotron motion and the wave number of the high mode and the low mode as a function of current. 


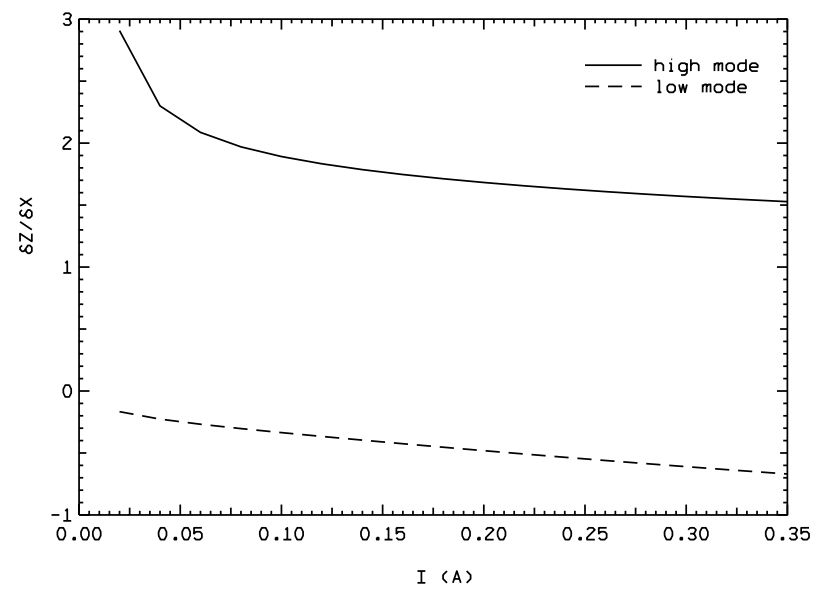

FIG. 5. The ratio of relative amplitude of longitudinal oscillation to that of transverse oscillation, $\delta R_{z} / \delta R_{x}$, for the high mode and the low mode as a function of current.

is predominantly transverse at low current and is out of phase with the longitudinal oscillation. Once again, the dominance decreases with increasing current.

The dominance of the longitudinal (transverse) oscillation in the high (low) mode is different from that observed in previous studies because, unlike previous studies, our physical parameters have a longitudinal focusing strength that is about the same as the transverse focusing strength at zero current $[12,17]$. Figure 6 shows the quantity $\delta R_{z} / \delta R_{x}$ as a function of the focusing strength ratio, $k_{z 0} / k_{x 0}$, with all the other physical parameters in Table I fixed. We see that, for the high mode, $\delta R_{z} / \delta R_{x}$ quickly decreases when $k_{z 0} / k_{x 0}<1$. Conversely, for the low mode, $\delta R_{z} / \delta R_{x}$ quickly increases (in absolute value) when $k_{z 0} / k_{x 0}<1$. This significantly changes the role that the low mode might play in the longitudinal beam halo dynamics. The ratio of longitudinal-to-transverse emittance in Table I is 2.05 .

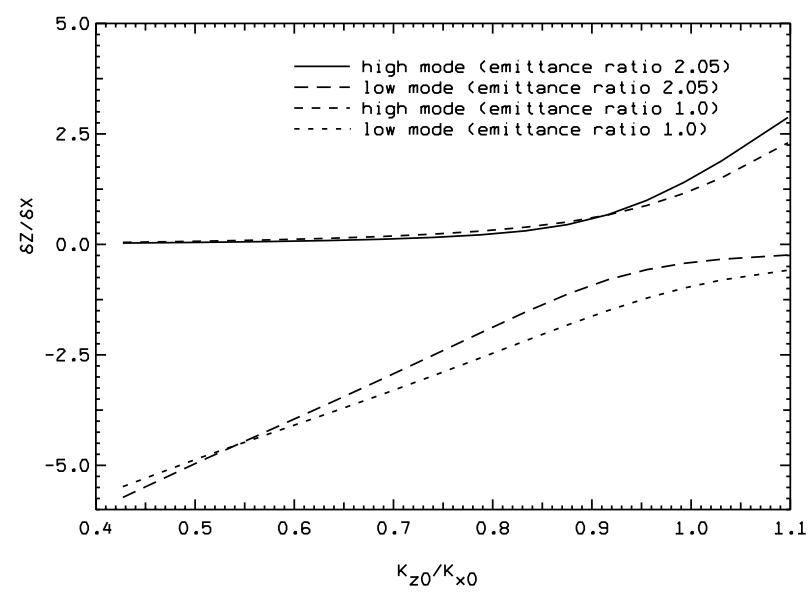

FIG. 6. The ratio of relative amplitude of longitudinal oscillation to that of transverse oscillation, $\delta R_{z} / \delta R_{x}$, as a function of the focusing strength ratio $k_{z 0} / k_{x 0}$.

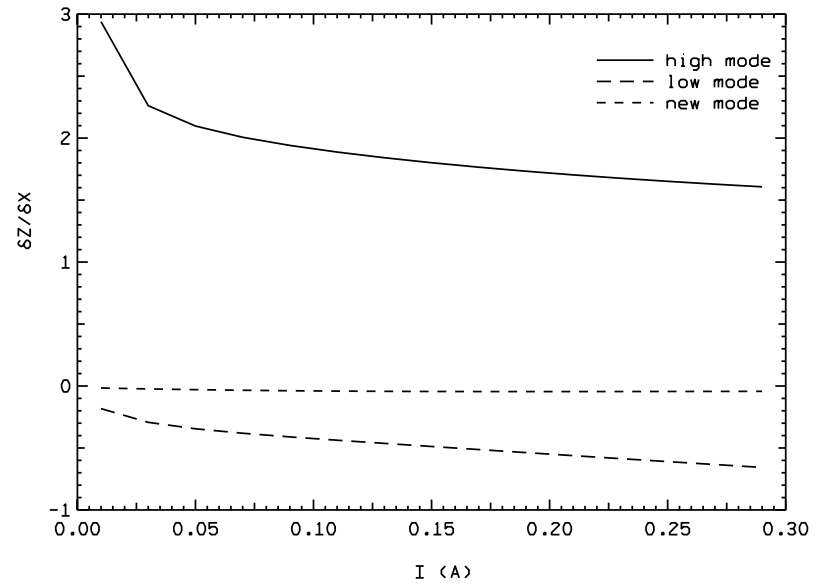

FIG. 7. The ratio of relative amplitude of longitudinal oscillation to that of transverse oscillation, $\delta R_{z} / \delta R_{x}$, as a function of the current for an ellipsoidal bunched beam without azimuthal symmetry.

To see the potential effects of emittance ratio on the characteristics of the envelope modes, we also show in Fig. 6 the relative oscillation amplitude, $\delta R_{z} / \delta R_{x}$, as a function focusing strength ratio for two values of emittance ratio, 2.05 and 1.0. It shows that, for these parameters, the relative oscillation amplitude is only weakly dependent on the emittance ratio.

In the above section, we discussed the properties of the linear envelope modes for a bunched beam with azimuthal symmetry. To study the effect of azimuthal asymmetry, we have also analyzed the modes using the parameters of Table I but with the $x$ emittance equal to 0.3 times the $y$ emittance shown in the table. For these parameters, the ratio of the $x$ size to $y$ size varies from 0.57 to 0.92 as current rises from 0.01 to $0.29 \mathrm{~A}$. Figure 7 shows the quantity $\delta R_{z} / \delta R_{x}$ of the three eigenmodes as a function of the current. Comparing with Fig. 5, we see that the high mode and the low mode exhibit a similar trend with and without azimuthal symmetry. However, the quadrupole mode from the azimuthally symmetric bunched beam no longer exists due to the presence of a longitudinal component. The phase of this longitudinal component depends on the ratio of longitudinal-to-transverse focusing strength and the ratio of $x$ to $y$ emittance.

\section{TEST PARTICLE DYNAMICS UNDER THREE ENVELOPE MODES}

A test particle in the particle-core model will feel both the external focusing force and the Coulomb force from the core. An initially matched core will have a constant radius in all three dimensions, and a test particle may move inside and outside of the core without a net change in energy. When the core is initially mismatched, the three eigenmodes can be excited. In this case, test particles starting 
from different initial phase space locations will have different patterns in phase space. When the ratio of test particle wave number to core envelope mode wave number is rational, a resonance between the test particle and core will be excited. Among these resonances, we are most interested in the 1:2 resonance. This is because this low order resonance will have a large oscillation amplitude and is generally found to be responsible for the generation of beam halo. The motion of a test particle under different envelope modes may show different behaviors. To understand the potential effects of these envelope modes on the test particle dynamics and beam halo formation, we use stroboscopic maps to study the test particle dynamics with only one envelope mode excited each time. The physical parameters are given in Table I. We have also considered only test particles with zero angular momentum. Figure 8(a) shows the stroboscopic plot in the $X-X^{\prime}$ plane under the high mode with a $20 \%$ initial transverse mismatch (and the corresponding 38\% longitudinal mismatch needed to excite the high mode and $6.7 \%$ longitudinal mismatch for the low mode). Figures 8(b) and 8(c) show analogous plots of the $X-X^{\prime}$ plane under the low mode and the quadrupole mode, respectively. In all the plots, we have strobed the test particle phase trajectory at the minimum of the horizontal oscillation of the core radius, i.e., the minimum of $\delta R_{x}$, since the extent of the resonant region in the figures is maximum at this point. Four regions are identified in Fig. 8. The first is the core region enclosed by the innermost circle that is determined by the envelope equation. The test particles initially starting in this region will be confined inside this region. The second region is between the core and the resonant region, and includes a separatrix. In the case of low current, the separatrix will confine particles. As the current increases, the separatrix will deteriorate and a stochastic sea will form around it [8]. The trajectories of particles starting from this region depend very sensitively on their initial conditions. These particles can move through the chaotic sea, at a rate governed by the maximum Lyapunov exponent, to large amplitude and to contribute to the formation of beam halo. The third region is the parametric resonance region. The test particles in this region will move to large amplitude due to the parametric resonance with core. The last region is the outermost region involving large-amplitude, betatronlike motion. Particles in this region will be part of the halo, but, in this model, particles cannot reach this region from elsewhere due to the presence of KolmogorovArnold-Moser curves.

The peanut structure on the $x-p_{x}$ plane suggests that the 1:2 resonance between test particles and the core envelope oscillation exists for all three modes. This is in agreement with the linear mode analysis in the last section for $100 \mathrm{~mA}$ beam current (see Fig. 3). Particles in the resonance region can move to very large amplitude to form halo particles. This will limit the minimum aperture that can be used in the accelerator design. For the
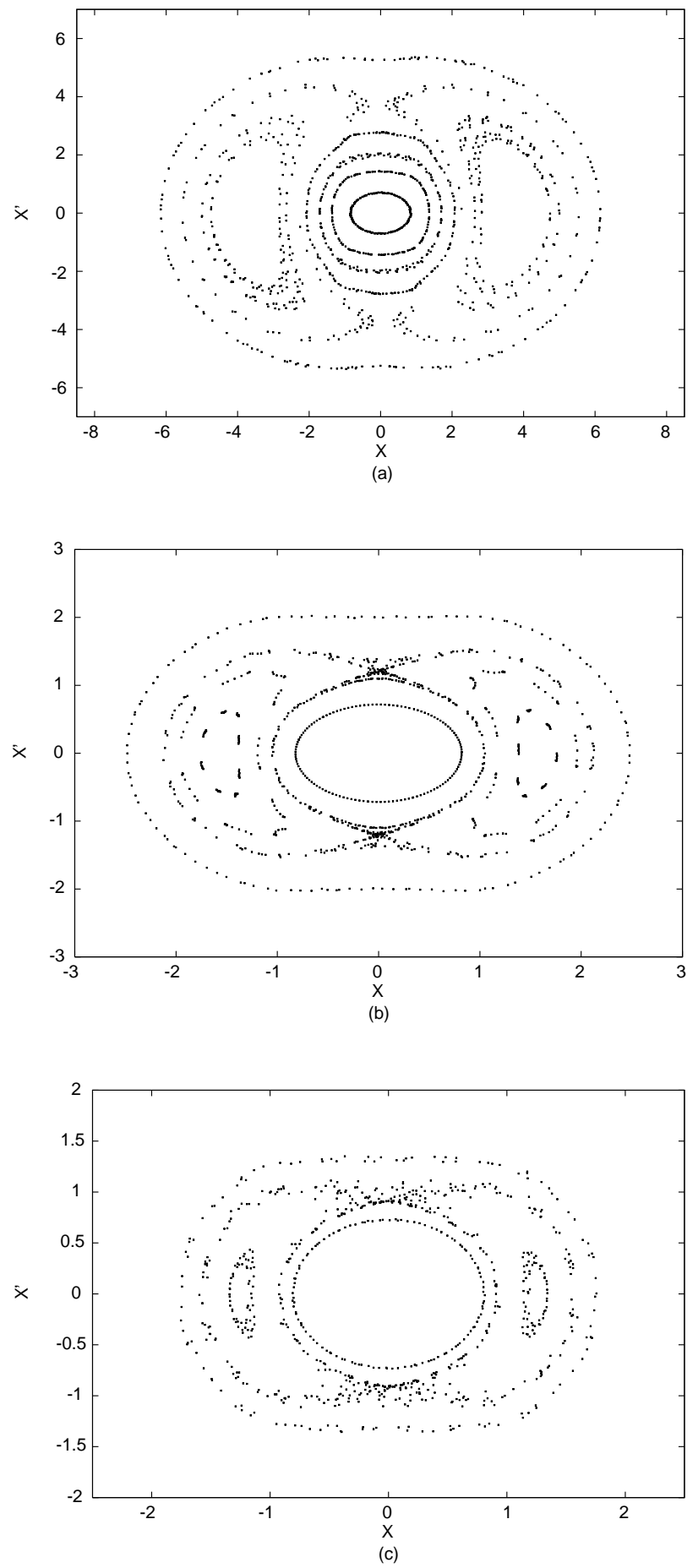

FIG. 8. (a) The stroboscopic plot in the $X-X^{\prime}$ plane under (a) the high mode, (b) the low mode, and (c) the quadrupole mode with a $20 \%$ initial transverse mismatch.

case of the high mode resonance, the outer edge of the 1:2 resonance is more than 5.0 units (i.e., 5 times the matched edge radius), which is much larger than the 1:2 resonance outer edges of the low mode and quadrupole mode. Thus, among the three resonances, the particlehigh mode resonance is the most serious in regard to 
maximum particle amplitude. On the other hand, the particle-high mode resonance also has the largest separation between the core and resonant regions in transverse phase space compared with the other modes. This means that, for high mode excitation, it will be more difficult for particles initially inside the core to reach the resonance region.

It is noteworthy that, for these parameters, the amplitude of the high mode resonance (which is 5 units) is larger than that which has been found in previous studies (typically 2-3 units for a spherical particle-core model; see, for example, Refs. $[12,13,17])$. Hofmann has pointed out that such large halo amplitudes are possible if the zero current longitudinal focusing strength is greater than the zero current transverse strength [25], as is the case of the present study. We have also verified that, for smaller transverse mismatches (as low as 5\%), the high mode amplitude is still approximately 4 units. However, as will be shown below, the high mode amplitude is greatly reduced (to approximately 2 units) as the focusing strength ratio approaches 0.6 , which is consistent with previous studies.
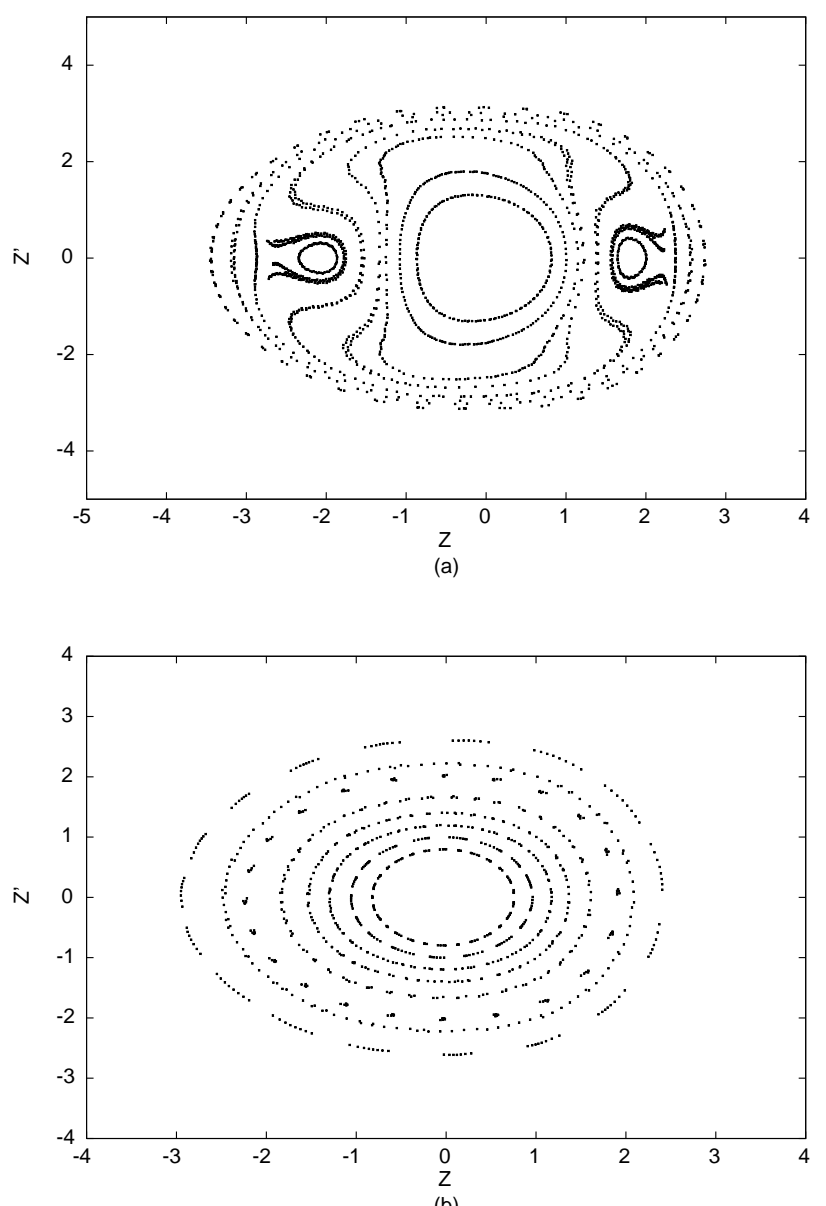

FIG. 9. The stroboscopic plot in the $Z-Z^{\prime}$ plane under (a) the high mode and (b) the low mode with a $20 \%$ initial transverse mismatch.
Turning to the quadrupole mode, this resonance is of the least concern with regard to maximum amplitude. In Fig. 8(c), the outer edge of the resonance is less than 2 units, which is the smallest among the three resonances. However, the separation between the core and the resonance is also smallest for the quadrupole mode. This suggests that particles initially inside the core are more likely to reach the resonance region [25].

In Figs. 9(a) and 9(b), we show stroboscopic plots of synchrotron motion in longitudinal phase space, $Z-Z^{\prime}$, for the low mode and the high mode. As mentioned previously, the rf nonlinearity causes the 1:2 resonance in the longitudinal plane to vanish in the low current regime [17]. This is why the 1:2 resonance appears only in the high mode [Fig. 9(a)]. Since the parametric resonance is a major mechanism of beam halo formation, this suggests that the high mode resonance will also be responsible for longitudinal beam halo formation under the physical parameters in Table I. The stroboscopic plots in Fig. 9(a) are not symmetric with respect to $Z=0$ due to nonlinear rf focusing effect. The 1:2 resonance between the particle and the high mode shows a somewhat different structure compared with the transverse resonance shown in Fig. 8(a). The resonant regions are smaller, and particles can move to larger amplitude before reaching the resonance region. The inner edge of the resonance extends to 1.6 units, and the outer edge reaches around 2.0 units.

From the analysis of the last section, the variation of the ratio of longitudinal focusing strength to transverse focusing strength can change the role played by the high mode and the low mode in longitudinal beam halo formation. The following shows test particle dynamics under three envelope modes with the focusing strength ratio equal to 0.634 and all the other physical parameters as shown in Table I. The stroboscopic plots of the transverse phase space for the high mode, low mode, and quadrupole mode are given in Figs. 10(a), 10(b), and 10(c), respectively, with 5\% initial transverse mismatch and $0.44 \%$ longitudinal mismatch for the high mode and $18 \%$ mismatch for the low mode. No 1:2 resonance is observed for the low mode in Fig. 10(b). The resonance extent for the quadrupole mode is about 1.5, which presents little danger for the machine design and operation. The outer edge of the high mode resonance in Fig. 10(a) reaches 2.1, which is more dangerous than the quadrupole mode resonance, but it is considerably smaller than the 5 unit amplitude seen in Fig. 8(a). Stroboscopic plots of the longitudinal phase space for the high mode and the low mode are given in Figs. 11(a) and 11(b), respectively. Here, the 1:2 resonance in the low mode has a maximum extent of 2.0 units. The 1:3 resonance is evident in the high mode. Comparing with the 1:2 resonance of the low mode, the 1:3 resonance has smaller amplitude and will be more difficult to populate by the particles initially inside 

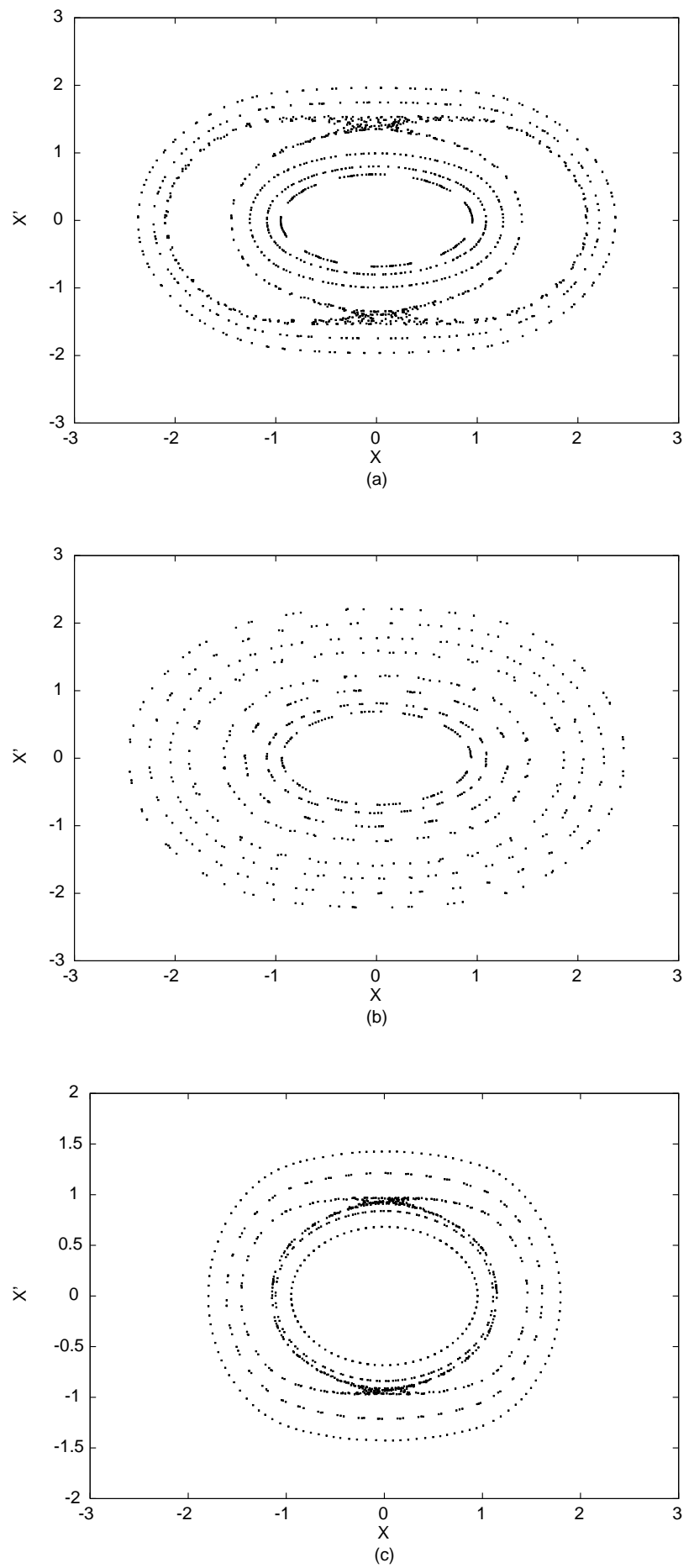

FIG. 10. The stroboscopic plot in the $X-X^{\prime}$ plane under (a) the high mode, (b) the low mode, and (c) the quadrupole mode with a 5\% initial transverse mismatch, with focusing strength ratio $k_{z 0} / k_{x 0}=0.634$.

the core. This suggests that, with a low ratio of longitudinal focusing to transverse focusing, the low-mode resonance will be a primary contributor to the formation of longitudinal halo.
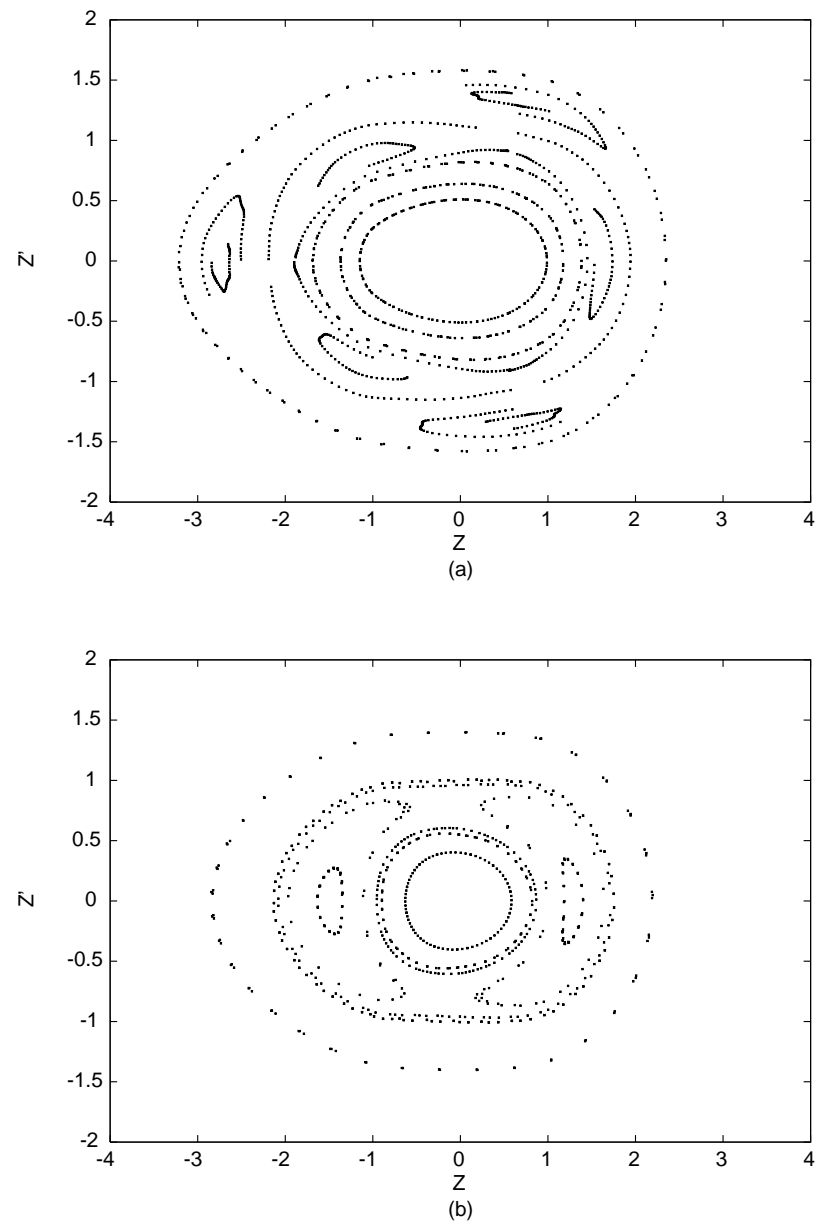

FIG. 11. The stroboscopic plot in the $Z-Z^{\prime}$ plane under (a) the high mode and (b) the low mode with a $5 \%$ initial transverse mismatch with focusing strength ratio $k_{z 0} / k_{x 0}=0.634$.

\section{CONCLUSIONS}

We have used a three-dimensional particle-core model to study particle dynamics in the presence of a mismatched ellipsoidal bunched beam and its impact on halo formation. Under the smooth approximation, we have used a linear transverse magnetic field to represent the average effect of an alternating gradient focusing channel. The nonlinear rf field in the rf gap is modeled by a continuous sinusoidal wave to represent the average effect of the synchronous rf wave, and acceleration through the rf gap is neglected. Three linear envelope modes, a high frequency mode, a low frequency mode, and a quadrupole mode, are identified for an azimuthally symmetric bunched beam. We have performed a systematic study of the features of the modes as a function of the longitudinal-to-transverse focusing strength ratio. We find that, as the ratio approaches unity, the oscillation of the high frequency mode contains a significant longitudinal component. The situation is reversed when the focusing strength ratio is sufficiently reduced, as has been the case in previous beam halo studies. Since the longitudinal 
and transverse focusing strengths are comparable in some designs of future high current linacs, this previously unrecognized property of the high frequency mode is an important factor for longitudinal beam halo formation. For the case of an ellipsoidal bunched beam without azimuthal symmetry, we find that the high frequency mode and the low mode are still present. However, the quadrupole mode is replaced by a new mode with transverse components $180^{\circ}$ out of phase and a nonzero longitudinal component. In addition to studying the mode properties as a function of focusing strength ratio, we have examined the features of the modes in phase space using stroboscopic plots. When the high frequency mode is excited, a 1:2 resonance between the mode and test particles is present in both the transverse and longitudinal phase spaces; when the low frequency mode is excited, the resonance is only present in the transverse phase space when the transverse to longitudinal focusing strength ratio is close to 1 . Also, the amplitude of the transverse resonance associated with the high mode is the largest of all three modes, making it the mode of greatest concern in regard to its impact on the size of the beam aperture. For the physical parameters given in Table I, the transverse amplitude of the high mode with a $20 \%$ initial transverse mismatch is roughly 5 times the matched beam size. By changing the parameters, we have observed even larger amplitudes. This large halo amplitude can have a significant impact on the design of high-intensity ion accelerators where the longitudinal-to-transverse focusing ratio is slightly greater than unity. Besides the issue of maximum particle amplitude, another important property of the modes is the separation between the core and the resonance region, since this has implications on the ability of particles initially in the core to reach the resonance. We find that, of all three modes, the quadrupole mode has the smallest such separation. Thus, though the quadrupole mode does not, by itself, lead to large amplitude particles, it may be the first step in a process, involving other modes, by which halo formation occurs.

\section{ACKNOWLEDGMENTS}

We are grateful to J. Barnard, K. Bongardt, P. Channell, A. Fedotov, R. Gluckstern, I. Hofmann, T. Wangler, and T.F. Wang for fruitful discussions. This work was performed on the SGI Origin 2000 at the Advanced Computing Laboratory located at Los Alamos National Laboratory and on the SGI/Cray T3E at the National Energy Research Scientific Computing Center located at Lawrence Berkeley National Laboratory. This work was supported by the DOE Grand Challenge in Computational
Accelerator Physics and the Los Alamos Accelerator Code Group.

[1] H. Koziol, Los Alamos MP Division Report No. MP-375-1, 1975.

[2] M. Reiser, C. Chang, D. Kehne, K. Low, T. Shea, H. Rudd, and J. Haber, Phys. Rev. Lett. 61, 2933 (1988).

[3] J. S. O'Connell, T.P. Wangler, R.S. Mills, and K. R. Crandall, in Proceedings of the 1993 Particle Accelerator Conference, Washington, DC, edited by S. T. Corneliussen (IEEE, Piscataway, NJ, 1993), p. 3657.

[4] R. L. Gluckstern, Phys. Rev. Lett. 73, 1247 (1994).

[5] R. A. Jameson, Los Alamos Report No. LA-UR-94-3753.

[6] J. Lagniel, Nucl. Instrum. Methods Phys. Res., Sect. A 345, 46 (1994).

[7] J. Lagniel, Nucl. Instrum. Methods Phys. Res., Sect. A 345, 405 (1994).

[8] T.P. Wangler, Los Alamos Report No. LA-UR-94-1135.

[9] C. Chen and R. A. Jameson, Phys. Rev. E 52, 3074 (1995).

[10] R. D. Ryne and S. Habib, Part. Accel. 55, 365 (1996).

[11] H. Okamoto and M. Ikegami, Phys. Rev. E 55, 4694 (1997).

[12] R. L. Gluckstern, A. V. Fedotov, S. S. Kurennoy, and R. D. Ryne, Phys. Rev. E 58, 4 (1998).

[13] T.P. Wangler, K. R. Crandall, R. Ryne, and T.S. Wang, Phys. Rev. ST Accel. Beams 1, 084201 (1998).

[14] A. V. Fedotov, R. L. Gluckstern, S. S. Kurennoy, and R. D. Ryne, Phys. Rev. ST Accel. Beams 2, 014201 (1999).

[15] M. Ikegami, Phys. Rev. E 59, 2330 (1999).

[16] T. F. Wang, Phys. Rev. E 61, 855 (2000).

[17] J. J. Barnard and S. M. Lund, in Proceedings of the 1997 Particle Accelerator Conference, Vancouver, Canada (IEEE, Piscataway, NJ, 1997), pp. 1929-1934; J. J. Barnard, S. M. Lund, and R.D. Ryne, in Proceedings of the XIX International Linear Accelerator Conference, Chicago, 1998 (IEEE, Piscataway, NJ, 1998), pp. 827829.

[18] I. Hofmann, J. Qiang, and R. D. Ryne, in Proceedings of the 1999 Particle Accelerator Conference, New York (IEEE, Piscataway, NJ, 1999), pp. 139-141.

[19] K. Bongardt, M. Pabst, and A. Letchford, in Proceedings of the XIX International Linear Accelerator Conference, Chicago, 1998 (Ref. [17]), pp. 824-826.

[20] N. Pichoff, CEA Report No. 91191, 1998.

[21] G. P. Lawrence, in Proceedings of the XIX International Linear Accelerator Conference, Chicago, 1998 (Ref. [17]).

[22] R. L. Gluckstern, W. Cheng, and H. Ye, Phys. Rev. Lett. 75, 2835 (1995).

[23] F. J. Sacherer, IEEE Trans. Nucl. Sci. 18, 1101 (1971).

[24] R. Ryne, Los Alamos Report No. LA-UR-95-391; http:// xxx.lanl.gov/abs/acc-phys/9502001.

[25] I. Hofmann (private communication). 